\title{
LINC00324 facilitates cell proliferation through competing for miR-214-5p in immature ovarian teratocarcinoma
}

\author{
MEILING CHEN $^{1}$, MIN ZHANG $^{2}$, LING XIE $^{3}$, SANSHAN WU $^{3}$ and YUANYUAN ZHONG ${ }^{4}$ \\ ${ }^{1}$ Gynecology Department, The First Hospital of Fuzhou Fujian, Fuzhou, Fujian 350009; \\ ${ }^{2}$ Department of Orthopedic Trauma, Yidu Central Hospital, Weifang, Shandong 261000; ${ }^{3}$ Department of Gynecology, \\ The First Hospital of Fuzhou Fujian, Fuzhou, Fujian 350009; ${ }^{4}$ Department of Obstetrics and Gynecology, \\ Wuhan Children's Hospital (Wuhan Maternal and Child Healthcare Hospital), Tongji Medical College, \\ Huazhong University of Science and Technology, Wuhan, Hubei 430013, P.R. China
}

Received September 27, 2019; Accepted March 26, 2020

DOI: $10.3892 /$ ijmm.2020.4800

\begin{abstract}
Immature ovarian teratocarcinoma (IOT) is a rare and malignant type of ovarian teratoma, and the molecular mechanisms underlying the pathogenesis and malignant phenotype of IOT remain uncharacterized. The present study examined a long non-coding RNA (lncRNA), long-chain intergenic non-coding RNA324 (LINC00324), which may serve a crucial role in pathogenesis of IOT. According to the results, LINC00324 was upregulated in IOT tissues and cells, as determined by reverse transcription-quantitative PCR, and its depletion impaired cell proliferation ability and improved cell apoptosis ability in IOT. Furthermore, LINC00324 acted as a miR-214-5p sponge to derepress cyclin dependent kinase 6 (CDK6), cyclin D1 (CCND1), murine double minute homolog 2 (MDM2), and mouse double minute 4 (MDM4) expression, thus increasing IOT cell proliferation and repressing apoptosis. Taken together, these results demonstrated that LINC00324 could serve as a competing endogenous RNA to facilitate IOT cell proliferation by regulation of miR-214-5p-CDK6/CCND1/MDM2/MDM4 network, which possibly provide a novel therapeutic target for IOT.
\end{abstract}

\section{Introduction}

Ovarian teratocarcinoma is a rare type of tumor, accounting for $\sim 30 \%$ of all types of ovarian cancer and originating from germ cell in the ovary (1). Histologically, ovarian teratocarcinoma

Correspondence to: Dr Yuanyuan Zhong, Department of Obstetrics and Gynecology, Wuhan Children's Hospital (Wuhan Maternal and Child Healthcare Hospital), Tongji Medical College, Huazhong University of Science and Technology, 100 Hong Kong Road, Jiang'an, Wuhan, Hubei 430013, P.R. China

E-mail: zhong82261267@126.com

Key words: long-chain intergenic non-coding RNA324, microRNA-214-5p, immature ovarian teratocarcinoma, proliferation, apoptosis can be classified into two subtypes, namely mature and immature types. Immature ovarian teratocarcinoma (IOT) is rare but aggressive, representing $1 \%$ of all teratomas and $1 \%$ of all ovarian neoplasms (2). It is also one of the most common histologic subtypes of malignant ovarian germ cell tumors (MOGCTs), representing $236.5 \%$ of MOGCT cases (3). Ovarian teratocarcinomas are mainly benign and can be cured by surgery, whereas IOT is a serious threat to the health of females at reproductive age (4). Further, the etiology of IOT remains elusive. Due to the rarity of IOT, relevant data and studies are limited and restricted to survival analyses of cases with rare experimental evidence.

Next-generation sequencing technology has revealed that majority of the human genome is transcribed into non-coding RNAs (ncRNAs), including microRNA (miRNA), circular RNA (circRNA), long noncoding RNA (lncRNA) and pseudogenes (5). These ncRNAs serve an essential role in gene regulation at the transcriptional and post-transcriptional levels (6), and their anomalous expression patterns are implicated in pathogenesis of multiple diseases, including cancer (7). The emergence of the 'competing endogenous RNA (ceRNA) hypothesis', which suggests that lncRNAs release target genes repressed by miRNAs via sequestering miRNAs, has attracted attentions from multiple researchers (8). To function as an effective miRNA decoy, the lncRNA usually primarily steadily expresses in cytoplasm and coexists in the RNA-induced silencing complex (RISC) with miRNA (9). For example, abnormally highly expressed lncRNA insulin growth factor 2 antisense in gastric cancer (GC) is predominantly localized in cytoplasm and serves as a ceRNA of miR-503 to promote the GC progression (10). Whether a similar regulatory pattern is implicated in IOT requires further exploration.

The present study aimed to identify key molecules that participate in the malignant phenotype of IOT and elucidate the underlying mechanism, and to provide new therapeutic targets for IOT.

\section{Materials and methods}

Clinical samples. A total of 45 paired IOT tissues and mature ovarian teratocarcinoma (MOT) (benign) tissues were obtained 
from patients (age range, 10-20 years) who underwent surgical resection at The First Hospital of Fuzhou Fujian between January 2014 and February 2019. Written consent to the usage of ovarian teratocarcinoma tissues in the present study were obtained from all patients (patients $\geq 18$ years old) or their legal guardians $(<18$ years old) prior to testing. The present study was approved by the Ethics Committee of The First Hospital of Fuzhou Fujian.

Cell culture and transfection. The human ovarian teratoma $\mathrm{Hs}$ 38.T cell line, 293T cell line and ovarian teratocarcinoma PA-1 cell line were commercially obtained from the American Type Culture Collection and cultured in Dulbecco's Modified Eagle Medium (DMEM; Sigma-Aldrich; Merck KGaA), followed by supplementation with $10 \%$ fetal bovine serum (Gibco; Thermo Fisher Scientific, Inc.) and $1 \%$ penicillin/streptomycin mix in a humidified atmosphere of $5 \% \mathrm{CO}_{2}$ at $37^{\circ} \mathrm{C}$. The full-length sequences of murine double minute homolog 2 (MDM2), mouse double minute 4 (MDM4), cyclin dependent kinase 6 (CDK6) and cyclin D1 (CCND1) were subcloned into a pcDNA3.1 vector (Addgene, Inc.) with an empty plasmid was used as a negative control, which was referred to as 'Vector'. miR-214-5p mimic and negative control (NC mimic) were purchased from Shanghai GenePharma Co., Ltd. A total of $50 \mathrm{nM}$ miR-214-5p mimic and $50 \mathrm{nM}$ NC mimic were separately transfected into cells. The short hairpin RNAs (shRNAs) were synthesized by Guangzhou Ribobio Co., Ltd. Similarly, shRNAs targeting LINC00324 (sh-LINC00324\#1, 5'-CCGGTAACC TATTCCTTGAAGACACCTCGAGGTGTCTTCAAGGAA TAGGTTATTTTTG-3'; sh-LINC00324\#2, 5'-CCGGTC ACATAATGTTGAAAGTCTGCTCGAGCAGACTTTCAA CATTATGTGATTTTTG-3'; and sh-LINC00324\#3, 5'-CCG GACAAATCTTAGACGTAATCCCCTCGAGGGGATTAC GTCTAAGATTTGTTTTTG-3') and negative control shRNA (sh-NC, 5'-CCGGTGCCACCTAAATTTACCAAGTCT CGAGACTTGGTAAATTTAGGTGGCATTTTTG-3') were also synthesized and obtained from Shanghai GenePharma Co., Ltd. The cells were transfected with $20 \mathrm{nM}$ shRNAs. The plasmids were transfected into cells in 6-well plates at a concentration of $2 \mu \mathrm{g}$ per well using Lipofectamine ${ }^{\circledR} 2000$ (Invitrogen; Thermo Fisher Scientific, Inc.).

RNA extraction and reverse transcription-quantitative $(R T-q P C R)$. Total RNA in the treated cells was extracted using a TRIzol ${ }^{\mathrm{TM}}$ Plus RNA Purification kit (Invitrogen; Thermo Fisher Scientific, Inc.). cDNA was transcribed using PrimeScriptTM II Reverse Transcriptase (Takara Biotechnology, Inc.). The temperature protocol for RT was as follows: $37^{\circ} \mathrm{C}$ for $15 \mathrm{~min}$ and $85^{\circ} \mathrm{C}$ for $5 \mathrm{sec}$. qPCR was conducted with SYBR ${ }^{\circledR}$ Premix Ex Taq ${ }^{\mathrm{TM}}$ II (Takara Bio, Inc.) in triplicate via iQ5 Real-time PCR detection system (Bio-Rad Laboratories, Inc.) following the manufacturer's protocol. The thermocycling conditions were as follows: $5 \mathrm{~min}$ at $95^{\circ} \mathrm{C}$, followed by 40 cycles of $95^{\circ} \mathrm{C}$ for $30 \mathrm{sec}$, annealing at $65^{\circ} \mathrm{C}$ for $45 \mathrm{sec}$, extension at $72^{\circ} \mathrm{C}$ for $30 \mathrm{sec}$, and a final extension of $72^{\circ} \mathrm{C}$ for 5 min. $\mathrm{U} 2$ and GAPDH were used as the endogenous controls. Relative expression of genes was analyzed using $2^{-\Delta \Delta \mathrm{Cq}}$ method (11). Primer sequences were as follows: miR-199a-5p forward, 5'-GCCGAGCCCAGTGTTCAGACT-3' and reverse, 5'-CCCAGTGTTCAGACTACCTGTTC-3'; miR-513c-5p forward, 5'-CCGAGTTCTCAAGGAGGTGTC-3' and reverse, 5'-TTC TCA AGGAGGTGTCGT TTAT-3'; miR-214-5p forward, 5'-TGAGTGCCTGTCTACACTTG-3' and reverse, 5'-TGCCTGTCTACACTTGCTGTGC-3'; miR-202-3p forward, 5'-TTATAGAGAGGTATAGGGCA-3' and reverse, 5'-AGAGGTATAGGGCATGGGAA-3'; LINC00324 forward, 5'-GGCCCCACAAATCACACAAC-3' and reverse, 5'-TAC CGACTTGGTGCCATTCC-3'; CDK6 forward, 5'-GCAGGG AAAGAAAAGTGCAATGA-3' and reverse, 5'-CCCGGA GATCGGTCTAGCTT-3'; MDM2 forward, 5'-GCGAGCTTG GCTGCTTCT-3' and reverse, 5'-TCCCTCAAGACTCCCCAG TT-3'; MDM4 forward, 5'-ATGATCAGCAGGAGCAGCAT-3' and reverse, 5'-GCTCTGAGGTAGGCAGTGTG-3'; CCND1 forward, 5'-AGGCTGTGTCCCTCTTCTCT-3' and reverse, 5'-GGTGGCACGTAAGACACACT-3'; GAPDH forward, 5'-GGAGCGAGATCCCTCCAAAAT-3' and reverse, 5'-GGC TGTTGTCATACTTCTCATGG-3'; and U6 forward, 5'-CTC GCTTCGGCAGCACA-3' and reverse, 5'-AACGCTTCACGA ATTTGCGT-3'.

Cell proliferation assays. For the Cell Counting Kit-8 (CCK-8) assay, the CCK-8 kit [Yeasen Biotechnology (Shanghai) Co., Ltd.] was utilized following the manufacturer's protocol. Treated PA-1 or Hs 38.T cells were seeded in the 96-well culture plates (1,000 cells/well). Absorbance at $450 \mathrm{~nm}$ was measured by a microplate reader (Multiskan ${ }^{\mathrm{TM}} \mathrm{MK} 3$; Thermo Fisher Scientific, Inc.) every $24 \mathrm{~h}$ during a 4 -day period. A colony formation assay was performed as described previously (12). An 5'-ethynyl-2'-deoxyuridine (EdU) assay was conducted using the Click-iT EdU Alexa Fluor 488 Imaging kit (Guangzhou RiboBio Co., Ltd.) according to manufacturer's protocol.

Flow cytometry analysis. For apoptotic analyses, treated PA-1 or Hs 38.T cells were double-stained with Annexin V-FITC (5 $\mu \mathrm{l})$ for $15 \mathrm{~min}$ at room temperature and propidium iodide (PI; $5 \mu \mathrm{g} / \mathrm{ml}$ ) for $15 \mathrm{~min}$ at $4^{\circ} \mathrm{C}$ and analyzed with a FACScan flow cytometer (BD Biosciences, Inc.). For cell cycle analyses, PA-1 cells were collected and fixed in $70 \%$ ethanol at $4^{\circ} \mathrm{C}$ overnight, then stained with $0.5 \mathrm{ml} \mathrm{FxCycle}{ }^{\mathrm{TM}} \mathrm{PI} / \mathrm{RNase}$ staining solution for $15 \mathrm{~min}$ at room temperature (Thermo Fisher Scientific, Inc.) and analyzed using a FACScan flow cytometer (Thermo Fisher Scientific, Inc.). Data were assessed with FlowJo software version 10.5.3 (FlowJo LLC).

Terminal-deoxynucleotidyl transferase mediated nick end labeling (TUNEL) assay. Cells were fixed with 4\% paraformaldehyde for $15 \mathrm{~min}$ at room temperature. Subsequently, cells were treated with $1 \mathrm{X}$ TUNEL reagent (Clontech Laboratories, Inc.) for $1 \mathrm{~h}$ at room temperature, and $10 \mathrm{mM}$ PBS was used as the washing buffer. The nuclei were stained with $1 \mathrm{X}$ DAPI for $10 \mathrm{~min}$ at room temperature. Images were captured using a fluorescence microscope (Olympus Corporation; magnification, $\mathrm{x} 100)$.

Subcellular fractionation assay. Cytoplasmic and nuclear RNA of the PA-1 cells were isolated by utilizing a Cytoplasmic \& Nuclear RNA Purification kit (Norgen) following the manufacturer's guidelines. $18 \mathrm{~S}$ and $\mathrm{U} 2$ served as the cytoplasmic and nuclear controls, respectively. 
Bioinformatics analysis. For differential miRNA analyses, miRNA sequence raw data were downloaded from Gene Expression Omnibus database (accession no. GSE98536; https://www.ncbi.nlm.nih.gov/geo/) and converted to fastaq format using SRAtoolkit. Raw data were processed according to previous study (13). Differentially expressed miRNAs between 2 MOGCT samples and 7 benign OGCT samples were analyzed by R-Language Limma Package (Gordon Smyth; v3.44.1; http://bioconductor.org/packages/release/bioc/html/limma.html) (14) with default setting and identified with $\mid \log 2 \mathrm{FCl}>1.0$ and $\mathrm{P}<0.05$ as the criteria. As for Kyoto Encyclopedia of Genes and Genomes pathway analyses, the signaling pathway analysis was performed using the DAVID database (v6.8; https://david.ncifcrf.gov/summary. jsp). IncRNAs that can bind with miR-214-5p were predicted using StarBase V3.0 (http://starbase.sysu.edu.cn/) by searching the miRNA-lncRNA column.

RNA fluorescence in situ hybridization (FISH). Fluorescein-labeled probes against long-chain intergenic non-coding RNA324 (LINC00324) and miR-214-5p were synthesized by Guangzhou RiboBio Co., Ltd. FISH was performed using the Fluorescence in situ Hybridization kit (Guangzhou RiboBio Co., Ltd.) following the manufacturer's protocol. Cells were stained with 1X DAPI for $10 \mathrm{~min}$ at room temperature, and fluorescence signals were captured using the LSM 900 laser scanning microscope (Zeiss AG; magnification, $\mathrm{x} 1,000)$.

RNA-binding protein immunoprecipitation (RIP). An RIP assay was performed using the Magna RIP ${ }^{\mathrm{TM}}$ RNA-binding Protein Immunoprecipitation kit (EMD Millipore). Anti-Ago2 (1:200; cat. no. MABE253; EMD Millipore) and anti-IgG antibodies (1:200; cat. no. AB21-KC; EMD Millipore) were used for immunoprecipitation. The co-precipitated RNAs were purified with $1 \mathrm{X}$ phenol-chloroform-isoamyl-alcohol mixture $(125: 24: 1 ; \mathrm{pH}<4)$, followed by $\mathrm{qPCR}$ to evaluate the enrichment of LINC00324 and miR-214-5p to Ago2.

RNA pull-down analysis. RNA pull-down was conducted as described previously (15). The biotinylated DNA probe complementary to LINC00324 was synthesized by Guangzhou RiboBio Co., Ltd. The level of miR-214-5p was analyzed by qPCR.

Luciferase reporter assay. The whole sequences of LINC00324, CDK6 3'UTR, CCND1 3'UTR, MDM2 3'UTR or MDM4 3'UTR were cloned into the pmirGLO vector (Promega Corporation). The mutant sequences were obtained using the KOD enzyme (Toyobo) and were cloned into pmirGLO vector (Promega Corporation) using T4 ligase (New England Biolabs, Inc.). 293T (Amercian Type Culture Collection) and PA-1 cells $\left(2 \times 10^{4}\right)$ were seeded into 24 -well plates, then transfected with plasmids, miR-214-5p mimic and controls using Lipofectamine ${ }^{\circledR} 2000$ (Invitrogen; Thermo Fisher Scientific, Inc.). A total of 2 days after transfection cells were harvested and subjected to luciferase activity analysis using DLR ${ }^{\mathrm{TM}}$ assay system (Promega Corporation), and the luciferase activity of each reporter was measured by normalizing to Renilla luciferase activity.
Western blot analysis. M-PER Mammalian Protein Extraction Reagent (Thermo Fisher Scientific, Inc.) was utilized to extract the total proteins from PA-1 or Hs 38.T cells. Protein concentration was measured by using a BCA kit (cat. no. 23227; Pierce; Thermo Fisher Scientific, Inc.). Following separation using $10 \%$ SDS-PAGE with $20 \mu \mathrm{g}$ protein per lane, PVDF membranes were blocked with $3 \%$ BSA in $1 \mathrm{X}$ TBST (Sigma-Aldrich; Merck $\mathrm{KGaA}$ ) at $37^{\circ} \mathrm{C}$ for $1 \mathrm{~h}$ and incubated overnight at $4^{\circ} \mathrm{C}$ with the following primary antibodies purchased from Abcam: Anti-Bcl-2 (1,000; cat. no. ab32124); anti-Bax (1,000; cat. no. ab32503); anti-Caspase-3 (1:1,000; cat. no. ab13847); anti-Cleaved Caspase-3 (1,000; cat. no. ab2302); anti-Caspase-9 (1,000; cat. no. ab32539); anti-Cleaved Caspase-9 (1,000; cat. no. ab2324); and anti-GAPDH (1,000; cat. no. ab9485). The membranes were then incubated with goat anti-mouse IgG H\&L conjugated to HRP (1:5,000; Abcam; cat. no. ab97040) for $4 \mathrm{~h}$ at $37^{\circ} \mathrm{C}$. An ECL detection kit (cat. no. 32134; Pierce; Thermo Fisher Scientific, Inc was adopted to detect the bands. The densitometric analysis was performed using Image $\mathbf{J}$ (v1.8.0; National Institutes of Health).

Statistical analysis. All data were analyzed using SPSS 22.0 software (IBM Corp.). Data obtained from at least 3 independent experiments are expressed as mean \pm standard deviation. A student's t-test and one-way analysis of variance were used to calculate significance. The Kaplan-Meier method was used to analyze of prognosis of patients with IOT. A Pearson's correlation test was used to analyze the correlation between LINC00324 and miR-214-5p, and between miR-214-5p and CDK6/CCND1/MDM2/MDM4 expression levels in IOT tissues. $\mathrm{P}<0.05$ was considered to indicate a statistically significant difference.

\section{Results}

miRNA-214-5p is downregulated in malignant ovarian teratocarcinoma and involved in the regulation of IOT cell viability and functions. Through analyzing miRNA expression profiling data (GSE98536) examined in a previous study (13), 4 miRNAs (miR-199a-5p, miR-513c-5p, miR-214-5p and miR-202-3p) were downregulated in 2 MOGCT samples obtained from two patients respectively diagnosed with dysgerma and primitive germ cell tumor compared with 7 benign ovarian germ cell tumor (OGCT) samples collected from 7 mature teratoma patients. As IOT is a common subtype of MOGCT, we hypothesized that these 4 miRNAs may have similar expression pattern in IOT tissues. Through conducting RT-qPCR in 45 paired MOT and IOT tissues, it was identified that that lower expression levels of miR-214-5p (0.231-fold change) and miR-202-3p (0.548-fold change) were exhibited in IOT tissues compared with MOT tissues (Fig. 1A). Moreover, low miR-214-5p expression was closely associated with shorter survival times in patients with ovarian teratocarcinoma (Fig. S1A). These 2 candidate miRNAs were selected for subsequent RT-qPCR validations in ovarian teratocarcinoma cells. As presented in Fig. 1B, miR-202-3p failed to exhibit differential expression between ovarian teratoma cells (Hs 38.T) and teratocarcinoma cells (PA-1), whereas that miR-214-5p was significantly downregulated in the PA-1 cells compared with the Hs 38.T cells. The functional differences of Hs 38.T and PA-1 cells 


\section{A}

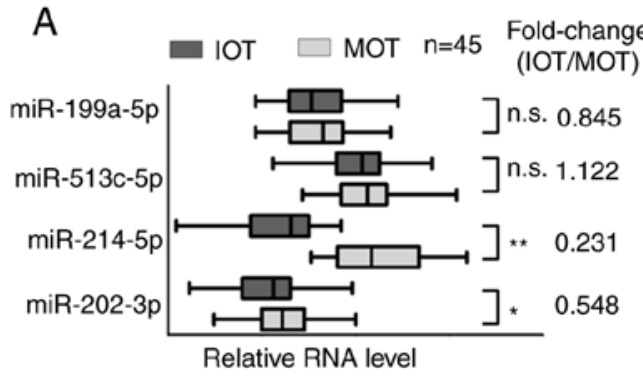

B

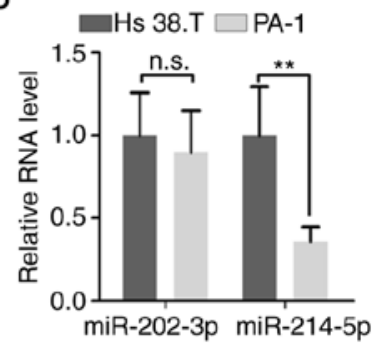

C

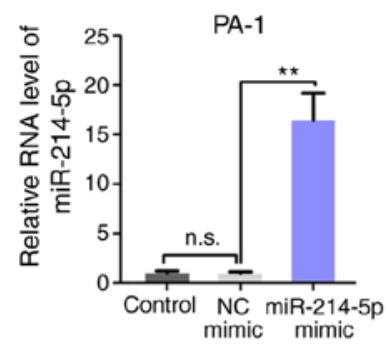

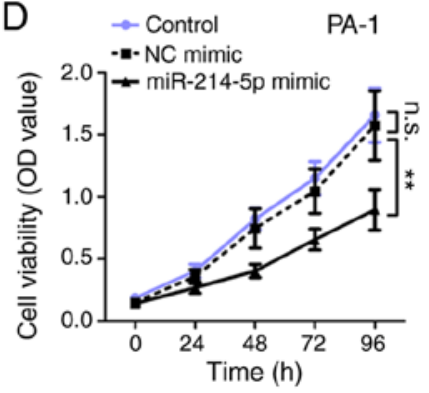

E

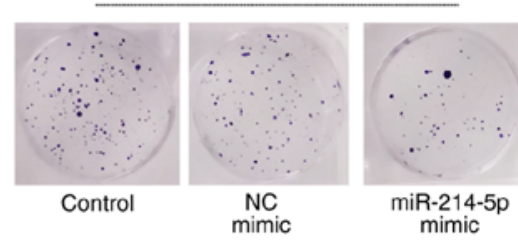

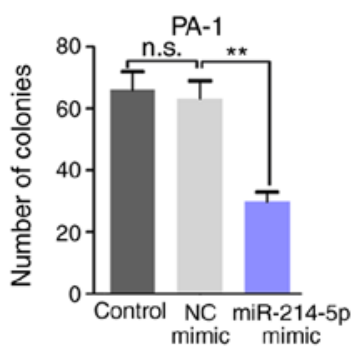
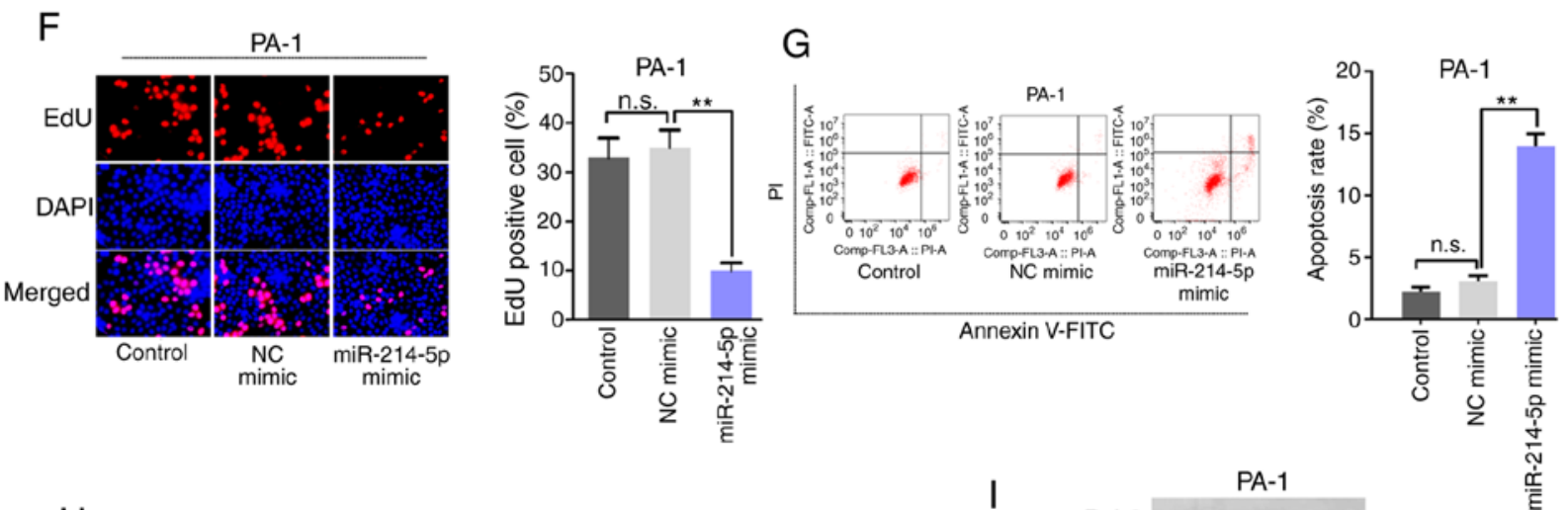

$\mathrm{H}$

PA-1

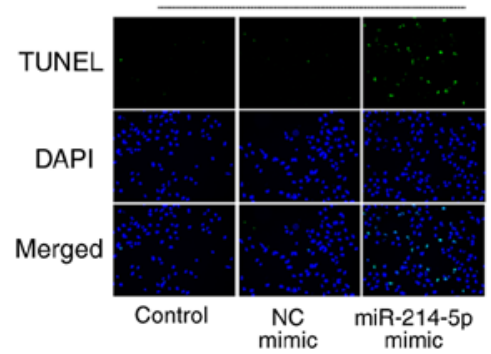

PA-1

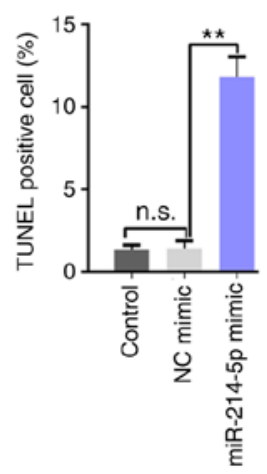

1

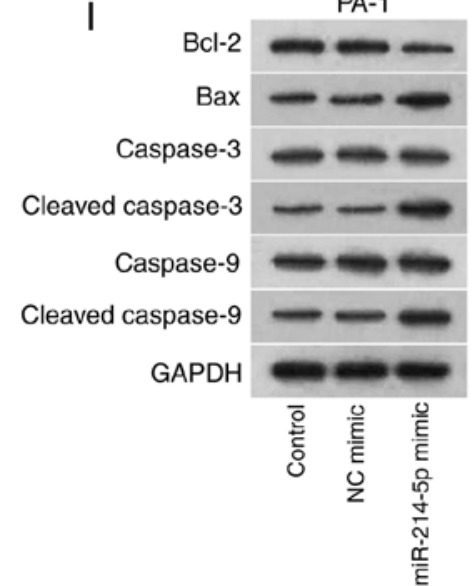

$\mathrm{PA}-1$

Figure 1. Expression profile and function of miR-214-5p in immature ovarian teratocarcinoma. (A) RT-qPCR analyzed the expression patterns of miR-199a-5p, miR-513c-5p, miR-214-5p, and miR-202-3p in 45 pairs of IOT and MOT tissues. (B) RT-qPCR determined the expression levels of miR-202-3p and miR-214-5p in Hs 38.T and PA-1 cells. (C) RT-qPCR determined the overexpression efficiency of miR-214-5p in PA-1 cells. (D) Cell Counting Kit-8 examined the cell viability in PA-1 cells. (E) Colony formation assay determined the clonogenic activity of PA-1 cells. (F) EdU assay determined PA-1 cell proliferation. Magnification, x200. (G) Flow cytometry analyzed the levels of apoptosis in PA-1 cells. (H) TUNEL assay determined the PA-1 cell apoptosis. Magnification, $\mathrm{x} 200$. (I) Western blot analysis examined changes in expression of the apoptosis-associated proteins. Control referred to untreated condition. ${ }^{*} \mathrm{P}<0.05$ and ${ }^{* *} \mathrm{P}<0.01$. n.s., no significance; miR, microRNA; RT-qPCR, reverse transcription-quantitative PCR; IOT, immature ovarian teratocarcinoma; MOT, mature ovarian teratocarcinoma; n.s., not significant; NC, negative control; OD, optical density; PI, propidium iodide; TUNEL, terminal-deoxynucleotidyl transferase mediated nick end labeling.

containing different expressions of miR-214-5p were also verified. As shown in Fig. S1B-D, CCK-8, colony formation and EdU assay data demonstrated that the proliferation ability of
Hs 38.T cells was inhibited compared with that of PA- 1 cells, as demonstrated by lower OD values, smaller colony numbers and a lower percentage of EdU-positive cells. Concomitantly, 
flow cytometry and TUNEL assays demonstrated that the apoptosis ability of Hs 38.T cells was improved compared with that of PA-1 cells, as demonstrated by the higher apoptotic cell rate and percentage of TUNEL-positive cells in Hs 38.T cells (Fig. S1E and F). In addition, the western blot analysis further examined the apoptosis ability of Hs 38.T and PA-1 cells. The expression level of anti-apoptosis protein (Bcl-2) was decreased in the Hs 38.T cells compared with the PA-1 cells. However, the pro-apoptosis proteins (Bax, Cleaved Caspase-3 and Cleaved Caspase-9) exhibited higher expression levels in Hs 38.T cells compared with the PA-1 cells (Fig. S1G). All these data supported the hypothesis that the downregulation of miR-214-5p may contribute to the malignancy of IOT.

Subsequently, gain-of-function assays were performed in PA-1 cells using a miR-214-5p mimic. A satisfactory overexpression efficiency of miR-214-5p was confirmed through RT-qPCR, which detected that the expression of miR-214-5p was increased in miR-214-5p mimic-transfected PA-1 cells compared with the NC mimic group (Fig. 1C). In a similar way, the results of CCK-8 assay demonstrated that miR-214-5p upregulation markedly attenuated PA-1 cell viability (Fig. 1D). In addition, the colony formation and EdU assays revealed that the colony number and percentages of the EdU-positive PA-1 cells decreased in response to miR-214-5p overexpression (Fig. 1E and F), further demonstrating that cell proliferation was inhibited by miR-214-5p upregulation. Moreover, the flow cytometry and TUNEL assay data suggested that overexpressing miR-214-5p induced the increased the apoptotic cell rate and the number of TUNEL-positive cells (Fig. $1 \mathrm{G}$ and $\mathrm{H}$ ). In concordance with the flow cytometry analyses data, the levels of Bax, cleaved caspase- 3 and cleaved caspase- 9 were upregulated upon overexpressing miR-214-5p, yet that of Bcl-2 was decreased in PA-1 cells (Fig. 1I). These data indicated that miR-214-5p overexpression inhibited cell proliferation and promoted cell apoptosis in ovarian teratocarcinoma.

Aberrantly expressed LINC00324 interacts with miR-214-5p and facilitates the malignant phenotype of IOT cells. Multiple studies have revealed that lncRNAs can interact with miRNAs via their miRNA response elements through ceRNA network (16). miR-214-5p was predicted to contain binding sites for 83 lncRNAs by StarBase V3.0 (http://starbase.sysu. edu.cn/). The present study then employed RT-qPCR to assess the expression profile of these 83 lncRNAs in randomly selected 3 paired IOT and MOT clinical samples. Among 29 aberrantly-expressed lncRNAs in the paired IOT and MOT tissue samples, 15 lncRNAs were upregulated in IOT tissues (Fig. 2A). LINC00324 was selected for subsequent analysis due to its most significant upregulation tendency $(\log F C=5.77$; $\mathrm{P}=0.002$ ). Moreover, Kaplan-Meier curve analysis indicated that high LINC00324 levels may be associated with unfavorable prognosis in patients with IOT (Fig. S2A). Then, RT-qPCR data demonstrated that LINC00324 was predominantly distributed in cytoplasmic fraction of PA-1 cells (Fig. S2B), indicating that LINC00324 may exert its function at post-transcriptional levels via ceRNA regulatory pattern. As miRNAs bind to their targets in an Ago2-dependent manner, an RIP assay was performed to verify their interaction using Ago2 antibody. It was identified that both LINC00324 and miR-214-5p could be co-immunoprecipitated by Ago2 antibody (Fig. 2B), indirectly suggesting the binding association between LINC00324 and miR-214-5p. The RNA pull-down assay measured the enrichment of miR-214-5p in the biotinylated LINC00324 wide-type (bio-LINC00324-Wt) group or the mutant form (bio-LINC00324-Mut) (Fig. 2C, upper), demonstrating that LINC00324 bound with miR-214-5p in a sequence-specific manner. Therefore, StarBase was used to predict the binding site between LINC00324 and miR-214-5p (Fig. 2C, lower panel). miR-214-5p was overexpressed in 293T cells (Fig. 2D). Additionally, the luciferase reporter assay indicated that the luciferase activity of LINC00324-Wt was repressed by the miR-214-5p mimic, and that of LINC00324-Mut was not affected (Fig. 2E), further confirming the binding association between LINC00324 and miR-214-5p. RNA-FISH demonstrated that LINC00324 and miR-214-5p co-localized in cytoplasm of PA-1 cells (Fig. S2C). The Pearson's correlation analysis data showed that LINC00324 was negatively correlated with miR-214-5p in IOT tissues (Fig. S2D). These data provided evidence that LINC00324 acted as a miR-214-5p sponge.

The role of LINC00324 in IOT cells was then investigated. The RT-qPCR data verified that LINC00324 was abnormally upregulated in ovarian teratocarcinoma PA-1 cells compared with the benign ovarian teratoma Hs 38.T cells (Fig. 2F). The efficiency of LINC00324 knockdown was examined for subsequent loss-of function assays. LINC00324 expression was decreased in sh-LINC00324\#1/2/3-transfected PA-1 cells (Fig. 2G). CCK-8, colony formation and EdU assays demonstrated that depleted LINC00324 inhibited PA-1 cell proliferation. When knocking down LINC00324, cell viability, colony number and EdU positive cells were all decreased (Fig. 2H-J). Furthermore, LINC00324 deficiency increased apoptosis in the PA-1 cells, which was verified by flow cytometry, TUNEL and western blot assays. Downregulating LINC00324 increased apoptotic cell rate and the number of TUNEL-positive cells. Additionally, pro-apoptosis protein expression was also elevated, and anti-apoptosis protein expression was suppressed in response to LINC00324 knockdown (Fig. 2K-M). Taken together, these data indicated that LINC00324 acted as a miR-214-5p sponge and promoted the malignant phenotype of IOT cells.

Tumor protein p53 (p53) signaling pathway is a downstream pathway of miR-214-5p. We further investigated whether the LINC00324/miR-214-5p axis affected IOT development via modulating signaling pathway. Through Kyoto Encyclopedia of Genes and Genomes pathway analyses, it was observed that the possible target genes of miR-214-5p, predicted by StarBase v3.0, were predominantly enriched in the p53 signaling pathway (Fig. 3A). Considering that lncRNAs could sponge miRNAs, thereby relieving the suppression of miRNAs on target genes in tumor cells, RT-qPCR was conducted to screen miR-214-5p target genes involved in the p53 pathway via knocking down LINC00324. As shown in Fig. 3B, 4 target genes (CDK6, MDM2, MDM4 and CCND1) were significantly downregulated in cells transfected with sh-LINC00324\#1/\#2/\#3 compared with controls. RIP suggested that the aforementioned mRNAs were immunoprecipitated by Ago2 antibody together with miR-214-5p, indicating that they co-existed in the RISC (Fig. 3C). The 


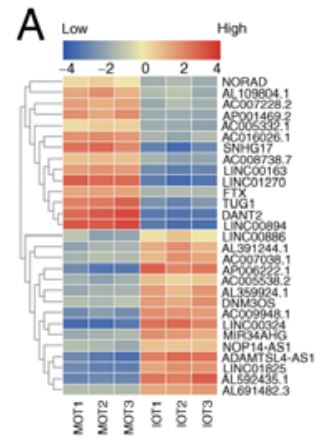

$\mathrm{E}$

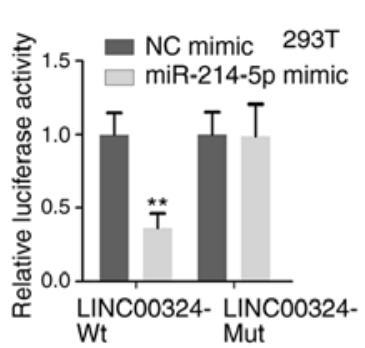

$\mathrm{H}$
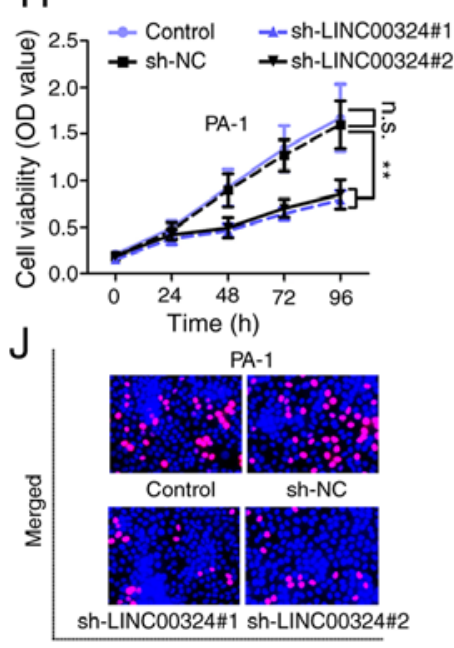

$\mathrm{L}$

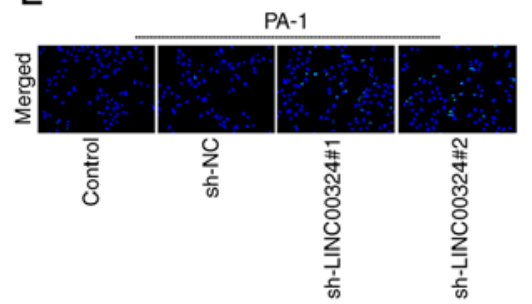

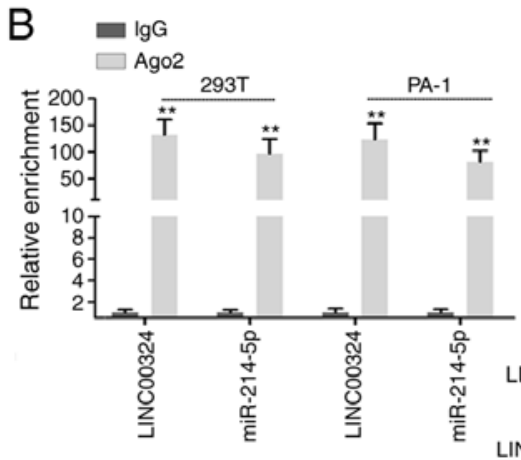

C $=$ Bio-NC

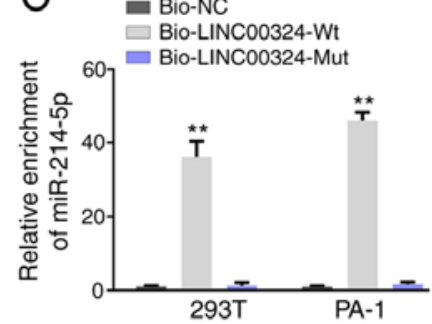

LINC00324-Wt: 5' ...aucaAGUCACGCUGUUGGACAGGCC...3'

miR-214-5p: $3^{\prime}$....cgugUC-GUUC-AC-AUCUGUCCGu...5

LINC00324-Mut: $5^{\prime}$...aucaAGUCACGCUGUUGCUGUCCGc...3'
$\mathrm{F}$

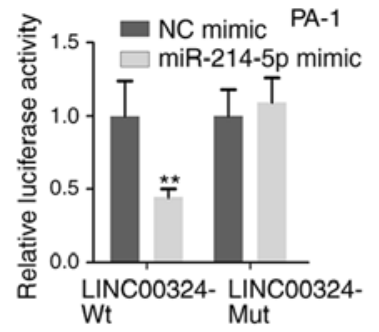

G

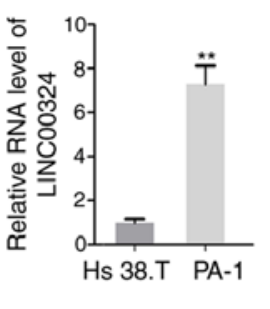

D

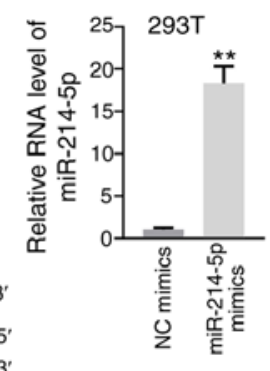

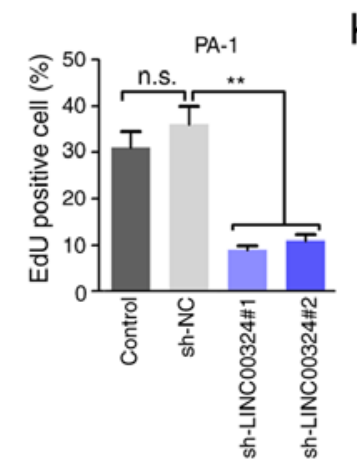

K

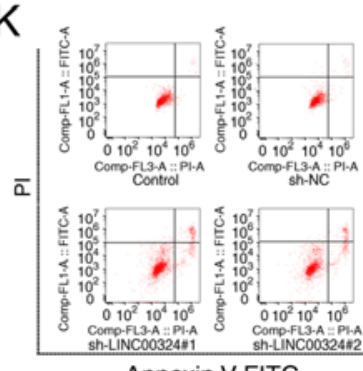

Annexin V-FITC
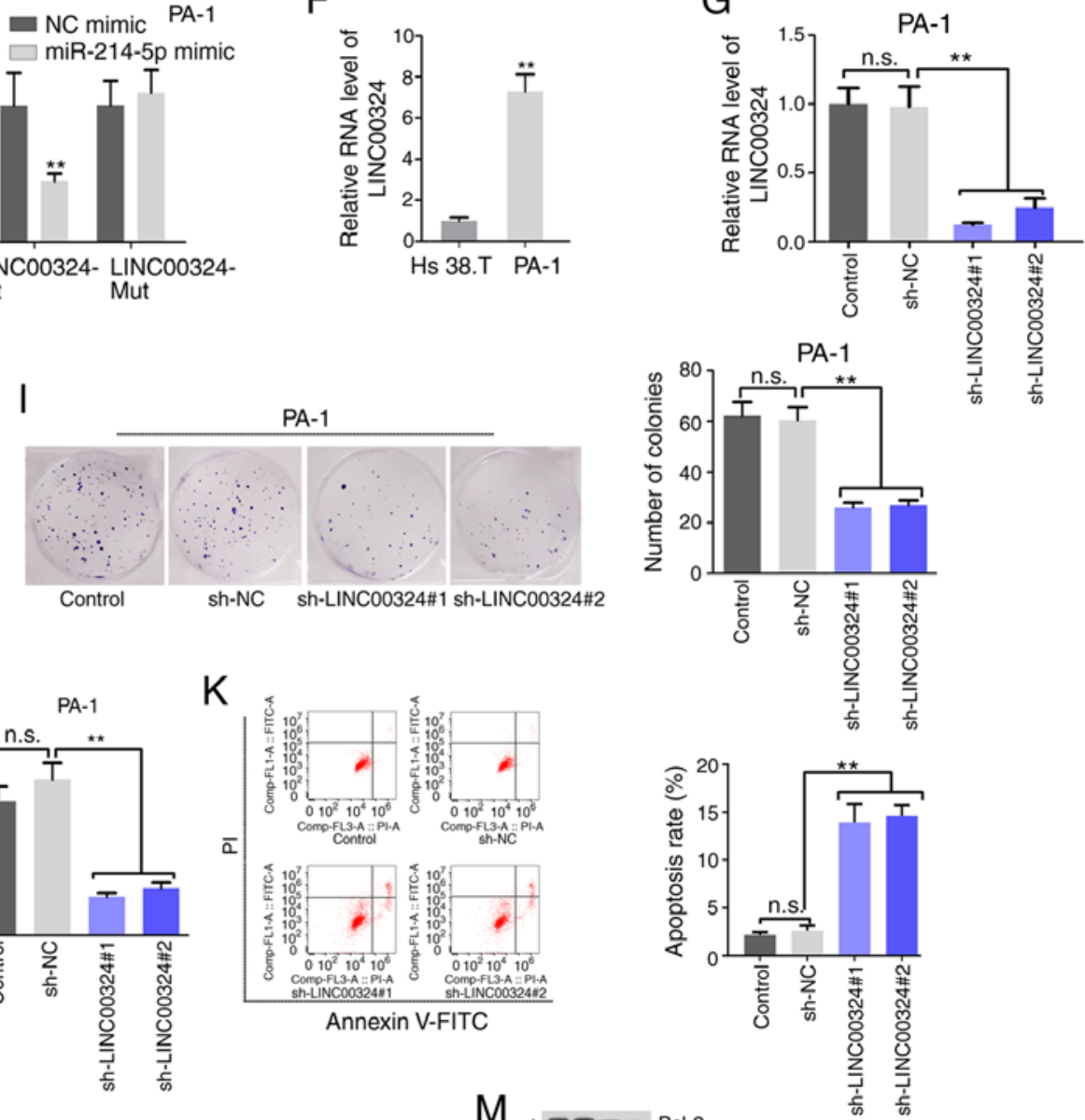

M

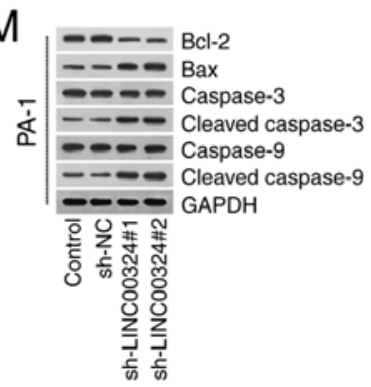

Figure 2. Aberrantly expressed LINC00324 functions as a miR-214-5p sponge and contributes to the malignant phenotype of PA-1 cells. (A) Hierarchical clustered heat map delineated the differentially expressed lncRNAs that possibly bind with miR-214-5p in 3 sets of paired MOT and IOT tissues, as examined by RT-qPCR. (B) RNA-binding protein immunoprecipitation with qPCR measured the enrichment of LINC00324 and miR-214-5p in Anti-Ago2 of 293T and PA-1 cells. Anti-IgG served as a negative control. (C) RNA pull-down measured the enrichment of miR-214-5p in Bio-LINC00324-Wt and Bio-LINC00324-Mut groups (upper panel). The binding sequence between LINC00324-Wt/LINC00324-Mut and miR-214-5p were indicated (lower panel). (D) RT-qPCR measured the overexpression efficiency of miR-214-5p in 293T cells. (E) A luciferase reporter assay examined the luciferase activity of LINC00324-Wt reporter and LINC00324-Mut reporter when overexpressing miR-214-5p. (F) RT-qPCR revealed the differential expression of LINC00324 in Hs $38 . T$ and PA-1 cells. (G) RT-qPCR examined the knockdown efficiency of LINC00324 in PA-1 cells. (H-J) The effect of LINC00324 knockdown on cell proliferation was analyzed by CCK-8, colony formation, and EdU assays. Magnification, x200. (K-M) The effect of LINC00324 knockdown on cell apoptosis was determined by (K) flow cytometry, (L) TUNEL assays and (M) western blot analysis. Magnification, $\mathrm{x} 200$. ${ }^{* *} \mathrm{P}<0.01$. LINC00324, long-chain intergenic non-coding RNA324; miR, microRNA; RT-qPCR, reverse transcription-quantitative PCR; Wt, wild-type; Mut, mutant; NC, negative control; n.s., not significant; sh, short hairpin; PI, propidium iodide; $\mathrm{OD}$, optical density. 
A
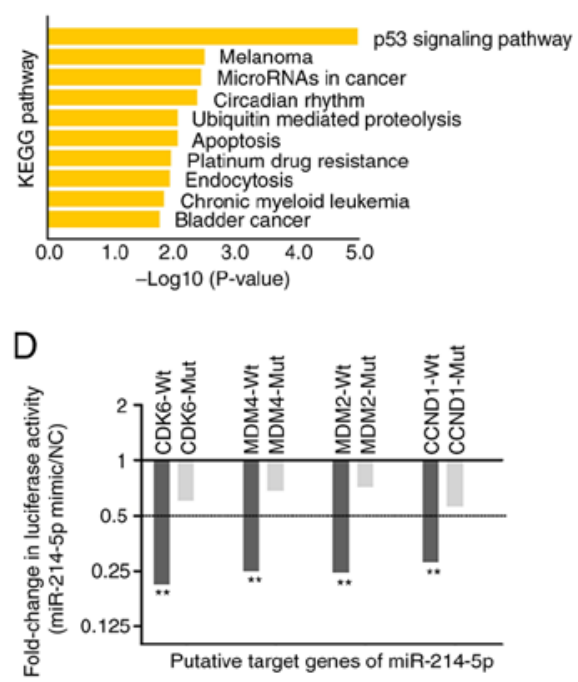

B L

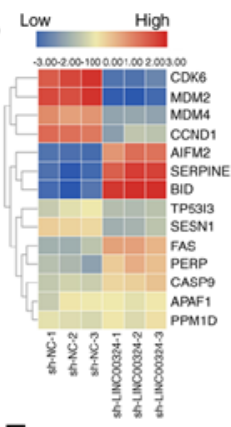

E

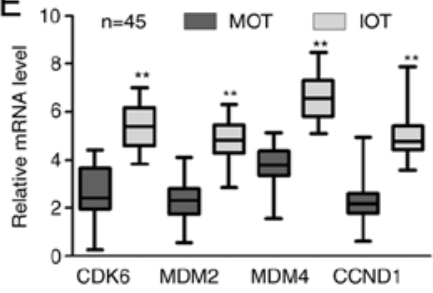

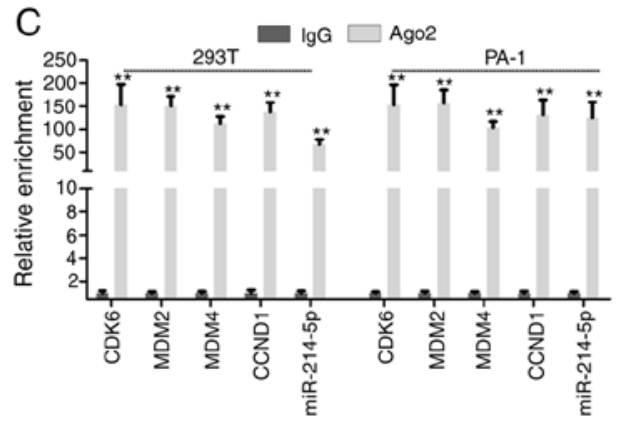

$\mathrm{F}$

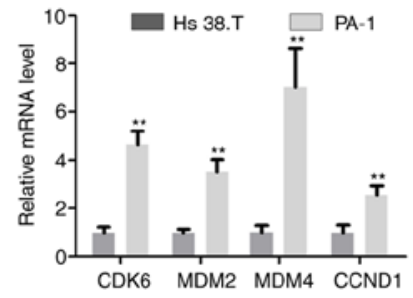

$\mathrm{G}$
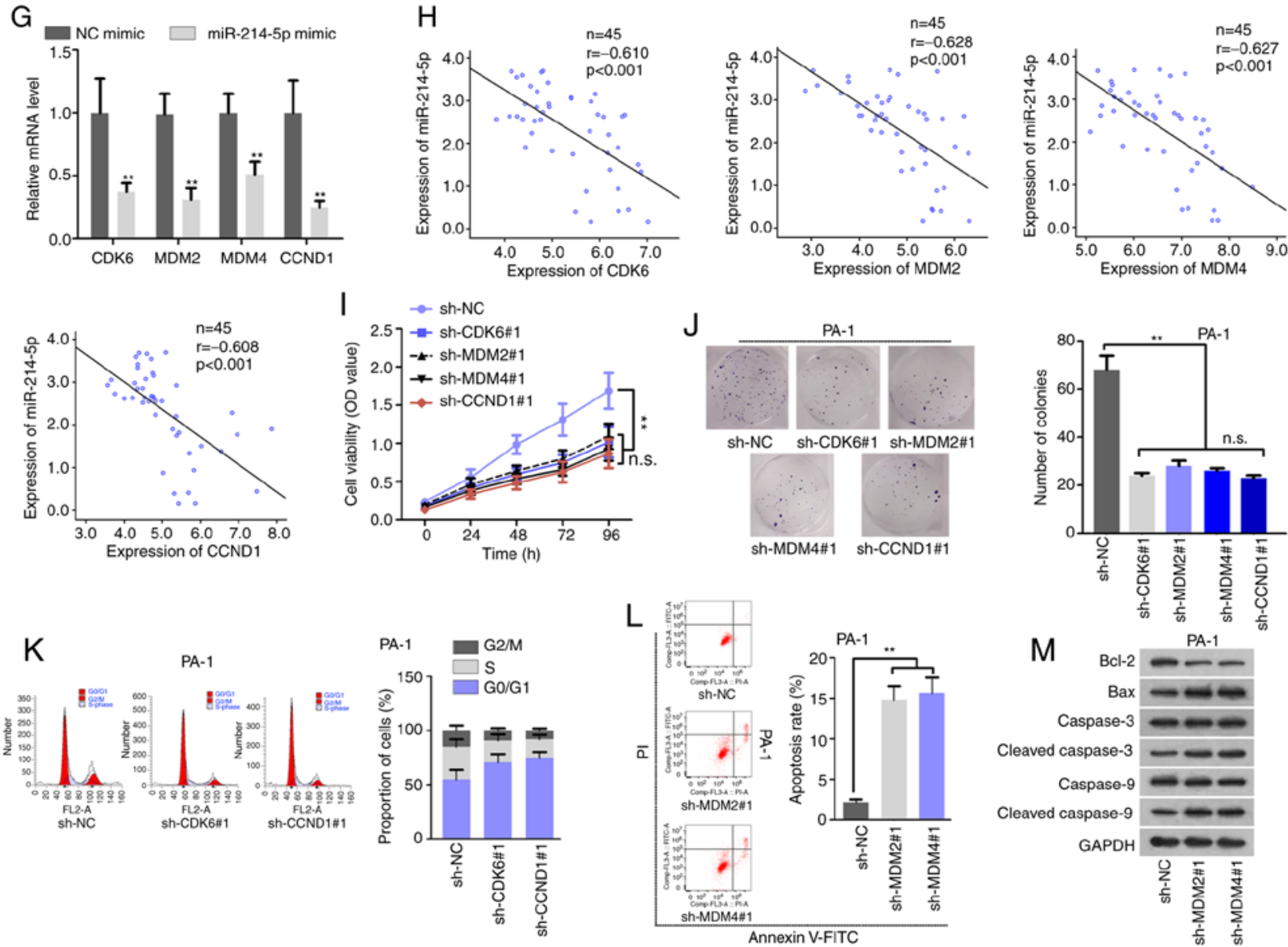

Figure 3. Downstream pathway of miR-214-5p was enriched in the p53 signaling pathway. (A) Kyoto Encyclopedia of Genes and Genomes pathway analyses predicted the potential target genes of miR-214-5p involved in several signaling pathways. (B) Hierarchical clustered heat map demonstrated the expression profile of miR-214-5p targets involved in the p53 pathway when knocking down LINC00324, as examined by RT-qPCR. (C) The RIP assay indicated that CDK6, MDM2, MDM4, CCND1 and miR-214-5p were immunoprecipitated by anti-Ago2 in 293T and PA-1 cells. (D) The luciferase reporter assay demonstrated a $>2$-fold change in the luciferase activity of CDK6/MDM4/MDM2/CCND1-Wt/Mut reporter following the overexpression miR-214-5p in PA-1 cells. (E) The expression patterns of CDK6, MDM2, MDM4 and CCND1 in 45 paired MOT and IOT tissues, as determined by RT-qPCR. (F) RT-qPCR determined the difference in expression levels of CDK6, MDM2, MDM4, and CCND1 in Hs 38.T and PA-1 cells. (G) RT-qPCR detected the effect of miR-214-5p overexpression on mRNA level of CDK6, MDM2, MDM4 and CCND1 in PA-1 cells. (H) Pearson's correlation analyses determined the correlations between CDK6/MDM2/MDM4/CCND1 and miR-214-5p in 45 IOT tissues. (I and J) The effect of interfering with CDK6, MDM2, MDM4 or CCND1 expression on PA-1 cell proliferation was assessed by (I) Cell Counting Kit-8 and (J) colony formation assays. (K) The effect of CDK6 or CCND1 knockdown on PA-1 cell cycle was analyzed by flow cytometry. (L and M) The effect of MDM2 or MDM4 knockdown on PA-1 cell apoptosis was analyzed by (L) flow cytometry and (M) western blot analysis. " $\mathrm{P}<0.01$. miR, microRNA; p53, tumor protein p53; LINC00324, long-chain intergenic non-coding RNA324; RT-qPCR, reverse transcription-quantitative polymerase chain reaction; CDK6, cyclin dependent kinase 6; MDM4, mouse double minute 4; MDM2, murine double minute homolog 2; CCND1, cyclin D1; Wt, wild-type; Mut, mutant; IOT, immature ovarian teratocarcinoma; MOT, mature ovarian teratocarcinoma; NC, negative control; OD, optical density; sh, short hairpin; n.s, not significant. 
putative binding sites of miR-214-5p on 3' untranslated regions (UTRs) of 4 mRNAs were indicated in Fig. S2E. The luciferase reporter assay data further confirmed that miR-214-5p was able to bind at sequence-specific sites within the 3'UTR of CDK6, MDM2, MDM4 and CCND1. The luciferase activity of the indicated mRNA wild types was markedly decreased compared with that of mRNA mutant types when overexpressing miR-214-5p (Fig. 3D). RT-qPCR analyses revealed that CDK6, MDM2, MDM4 and CCND1 mRNA levels were upregulated in malignant ovarian teratocarcinoma tissues and cells (Fig. 3E and F), and were suppressed by introduction of exogenous miR-214-5p (Fig. 3G). Moreover, Kaplan-Meier analyses demonstrated that the high levels of CDK6, MDM2, MDM4 and CCND1 were associated with the poorer prognosis of patients with IOT (Fig. S2F). Pearson's correlation analyses demonstrated that miR-214-5p was negatively correlated with CDK6, MDM2, MDM4 and CCND1 (Fig. 3H). To explore the cellular functions of these mRNAs, RT-qPCR and western blot analysis were conducted to knock down them using 2 shRNAs, respectively, and selected ones that demonstrated the best knockdown efficiency. The results indicated that the mRNA and protein levels of CDK6, MDM2, MDM4 and CCND1 were suppressed. Besides, the knockdown efficiency of sh-CDK6\#1, sh-MDM2\#1, sh-MDM4\#1 and sh-CCND1\#1 was detected by comparing with sh-NC (Fig. S3A-E). The CCK-8 and colony formation assays subsequently revealed that depletion of CDK6, MDM2, MDM4 and CCND1 could decrease the rate of cell proliferation (Fig. 3I and J). A previous study identified that CCND1 could regulate the cell cycle together with CDK6 (17). It has been also demonstrated that MDM2/MDM4 proteins exacerbate the interaction with p53 in tumor cells to attenuate apoptotic activity of p53 $(18,19)$. The effects of CCND1/CDK6 deficiency on cell cycle and MDM2/MDM4 deficiency on cell apoptosis were then explored. Flow cytometry analysis indicated that depletion of CCND1 or CDK6 resulted in $\mathrm{G} 1$ cell cycle arrest (Fig. 3K). In addition, MDM2 or MDM4 insufficiency accelerated cell apoptosis, which was validated by flow cytometry and western blot analysis data (Fig. 3L and M). Taken together, the results suggest that the downstream targets (CDK6/CCND1/MDM2/MDM4) of miR-214-5p may promote cell proliferation via regulation of cell cycle and apoptosis.

Abnormal proliferation of IOT cells is regulated by the LINC00324-miR-214-5p-CDK6/CCNDI/MDM2/MDM4ceRNA network. Whether LINC00324 affected biological function of IOT cells via the miR-214-5p-CDK6/CCND1/MDM2/MDM4 axis remains unknown. Therefore, a series of rescue assays were conducted using overexpression CDK6, CCND1, MDM2 and MDM4. Prior to that, the overexpression efficiency of these mRNAs was analyzed by RT-qPCR and western blot analysis, and vector was used as the negative control (Fig. S3F-J). In the rescue assays, the upregulation of CDK6, CCND1, MDM2 or MDM4 counteracted the suppressive effects of LINC00324 knockdown on cell proliferation (Fig. 4A-C). In addition, CDK6 or CCND1 overexpression reversed the LINC00324 knockdown-mediated function on cell cycle arrest in PA-1 cells (Fig. 4D). Additionally, MDM2 or MDM4 overexpression repressed cell apoptosis, originally activated by LINC00324 downregulation (Fig. 4E and F). Finally, the western blot assay data further confirmed the adverse role of MDM2 or MDM4 overexpression on sh-LINC00324-stimulated cell apoptosis (Fig. 4G). These data demonstrated that LINC00324 accelerated cell proliferation and inhibited cell apoptosis in IOT via sponging miR-214-5p to upregulate CDK6/CCND1/MDM2/MDM4 expression (Fig. S4).

\section{Discussion}

Over the previous decade, an increasing number of studies have elucidated that dysregulated miRNAs are implicated in the initiation and progression of human malignancies. Liu et al (20) uncovered that miR-34a was expressed at low levels in CD44 prostate cancer and its ectopic expression suppressed clonogenic activity and metastasis of tumor cells (20). miRNA sequencing (miRNA-seq) is a useful method for the identification of differentially expressed miRNAs. Zhu et al (15) performed miRNA-seq analyses in non-epithelial ovarian tumors and identified that miR-199a-5p, miR-214-5p, miR-513c-5p and miR-202-3p were downregulated in malignant ovarian germ cell tumors. Although the MOGCT samples analyzed by Zhu et al (15) did not involve IOT samples, we hypothesized that expression of these miRNAs was also dysregulated in IOT samples. Using an RT-qPCR assay, the present study identified that only miR-214-5p was downregulated in the IOT tissues and cells. The high level of miR-214-5p contributed to the prognosis of the patients of IOT. miR-214-5p is one of the mature isoforms of miR-214, which are implicated in the pathogenesis of multiple cardiovascular diseases (21). Previously published studies have also revealed that miR-214-5p serves a tumor-inhibitor role in various types of cancer, such as prostate cancer (22), hepatocellular carcinoma (23) and pancreatic cancer (24). To the best of our knowledge, the present study is the first to reveal the functions of miR-214-5p in IOT, which may provide a promising therapeutic target for IOT.

LINC00324, also known as C17orf44, a 2,115-bp long intervening/intergenic noncoding RNA located on human chromosome $17 \mathrm{p} 13.1$, has been demonstrated to serve an oncogenic role in various types of cancer. Wu et al (25) and Pan et al (26) identified that LINC00324 inhibited cell apoptosis and facilitated cell proliferation and migration in osteosarcoma and lung adenocarcinoma $(25,26)$. In addition, Zou et al (27) demonstrated that LINC00324 exerted carcinogenic functions in gastric cancer. In the present study, it was first verified that LINC00324 was upregulated in IOT tissues and cells. Besides, high expression of LINC00324 was closely associated with poor prognosis in patients with IOT. LINC00324 served as the miR-214-5p sponge in IOT cells. In addition, LINC00324 silencing suppressed cell proliferation and encouraged cell apoptosis in IOT, indicating that LINC00324 was an oncogenic gene in IOT and may be a potential therapeutic target of IOT.

The present study also suggested that LINC00324 knockdown apparently led to a decrease in CDK6, CCND1, MDM2 and MDM4 expression levels. CDK6, CCND1, MDM2 and MDM4 expression levels were upregulated in IOT tissues, and were negatively correlated with miR-214-5p. Dysregulation of these mRNAs was also closely associated with poor prognosis in patients with IOT. Moreover, CDK6 can form cyclin D-CDK4/6 complexes in the control of cell cycle G1 


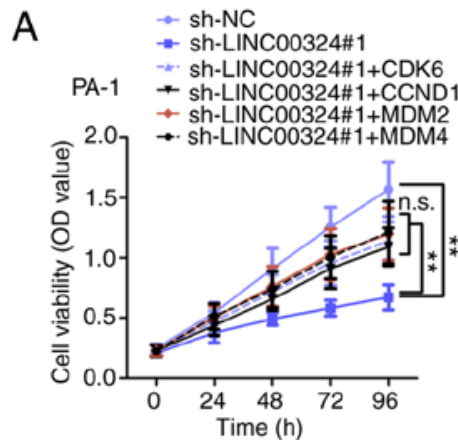

C

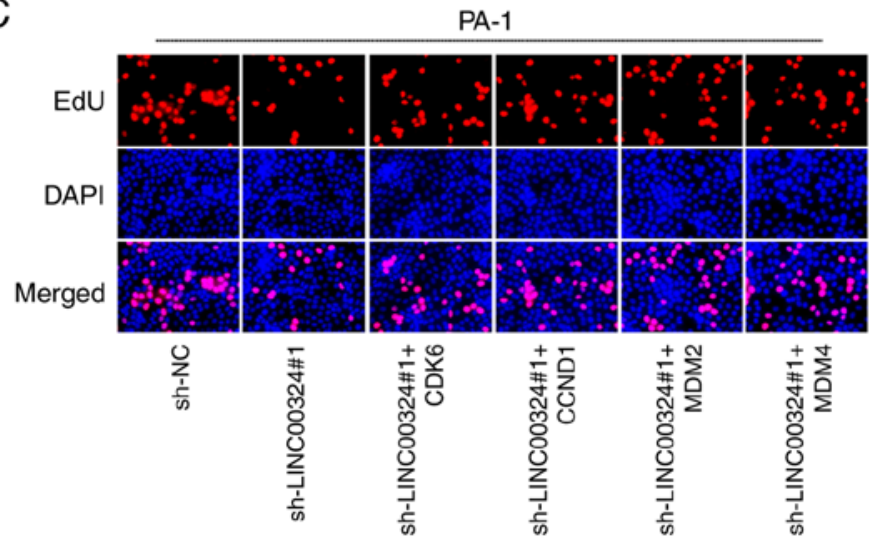

B

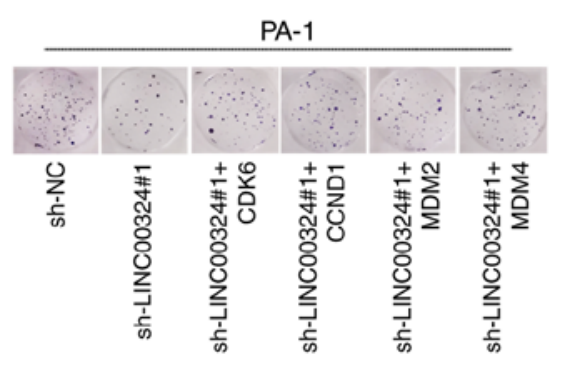

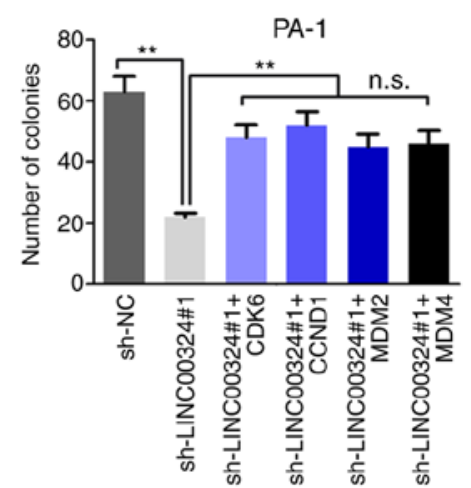

PA-1

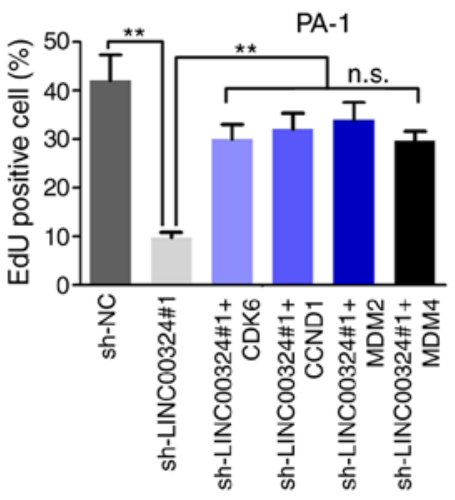

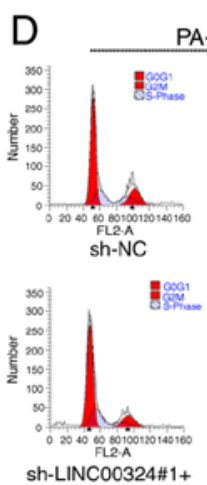

sh-LINC00324\# 1+

CDK6
PA-1
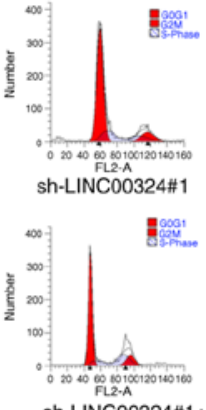

sh-LINC00324\#1 CCND1
F

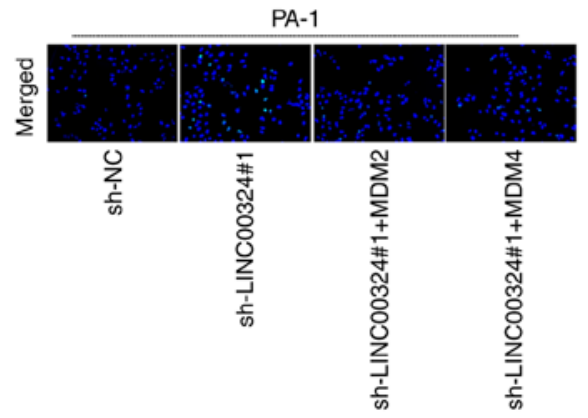

E

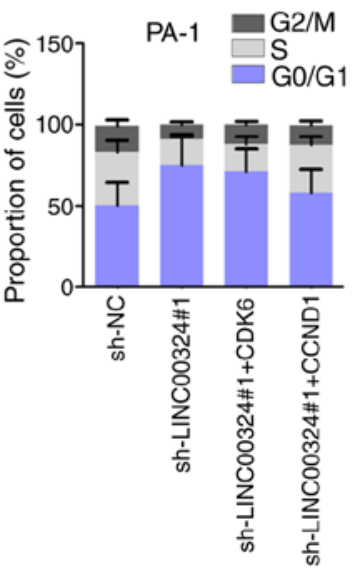

PA-1
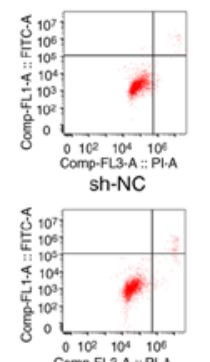

sh-LINC00324\#1

MDM2

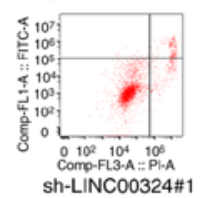

Sh-LINCO0324\#1

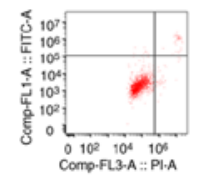

sh-LINCOO324\#1+

MDM4

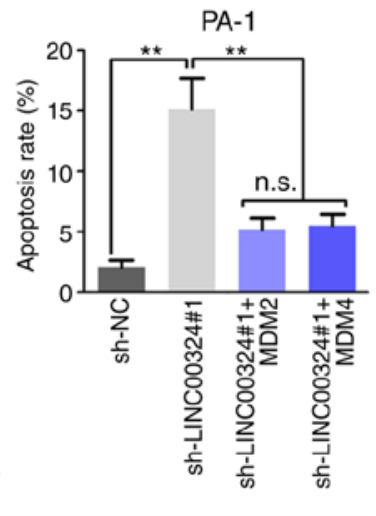

Annexin V-FITC

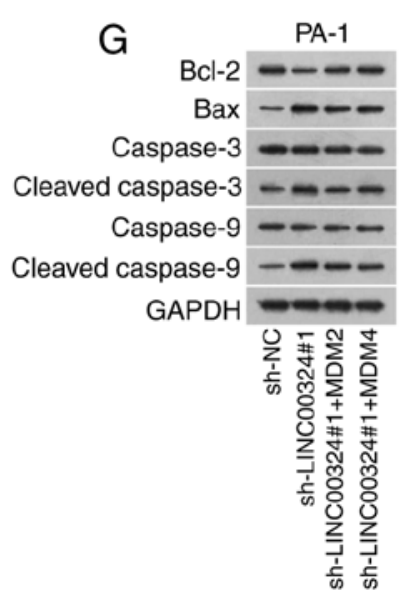

Figure 4. Abnormal proliferation of IOT cells was regulated by LINC00324-miR-214-5p-CDK6/CCND1/MDM2/MDM4 ceRNA network. (A) CCK-8, (B) colony formation and (C) EdU assays measured cell proliferation in different transfection groups. Magnification, x200. (D) Flow cytometry analyses measured G1 cell cycle situation in different transfection groups. (E) Flow cytometry, (F) TUNEL and (G) western blot analysis evaluated the levels of cell apoptosis in differently transfected cells. Magnification, $\mathrm{x} 200 .{ }^{* *} \mathrm{P}<0.01$. IOT, immature ovarian teratocarcinoma; LINC00324, long-chain intergenic non-coding RNA324; miR, microRNA; CDK6, cyclin dependent kinase 6; MDM4, mouse double minute 4; MDM2, murine double minute homolog 2; CCND1, cyclin D1; ceRNA, competing endogenous RNA; sh, short hairpin; NC, negative control; OD optical density; PI, propidium iodide; n.s, not significant. 
phase progression and G1/S transition (28). CCND1 encodes cyclin D1 and forms a complex with CDK6 to regulate CDK6 activity (17,29). The homologs MDM2 and MDM4 also form a MDM2/MDM4 complex that binds with p53 to suppressing p53 transcriptional activity, ultimately resulting in p53 proteasomal degradation and an inhibition of cell apoptosis (30). In the present study, knockdown of CDK6 or CCND1 induced G1 cell cycle arrest in IOT. CDK6 or CCND1 overexpression may mitigate the LINC00324 knockdown induced cell cycle arrest. Furthermore, the depletion of MDM2 or MDM4 promoted cell apoptosis, and ectopic upregulation of MDM2 or MDM4 may offset the stimulating function of LINC00324 efficiency on cell apoptosis. However, whether LINC00324 regulates IOT malignant phenotype through affecting the formation of CDK6/CCND1 or MDM2/MDM4 requires further exploration.

In summary, LINC00324 facilitates cell proliferation through competitively binding with miR-214-5p to upregulate CDK6, CCND1, MDM2 and MDM4 expression levels under the suppression of miR-214-5p in IOT cells.

\section{Acknowledgements}

Not applicable.

\section{Funding}

No funding was received.

\section{Availability of data and materials}

The datasets used and/or analyzed during the current study are available from the corresponding author on reasonable request.

\section{Authors' contributions}

MC conceived the study, analyzed the data and drafted the manuscript. YZ and MZ helped design the experiments. LX and SW helped collect data and design the study. All authors read and approved the final version of the manuscript.

\section{Ethics approval and consent to participate}

Written informed consent for the use of ovarian teratocarcinoma tissues in the present study were obtained from all patients or their legal guardians prior to testing. The study was approved by the Ethics Committee of The First Hospital of Fuzhou Fujian (Fuzhou, China).

\section{Patient consent for publication}

Not applicable.

\section{Competing interests}

The authors declare that they have no competing interests.

\section{References}

1. Outwater EK, Siegelman ES and Hunt J:L: Ovarian teratomas: Tumor types and imaging characteristics. Radiographics 21: 475-490, 2001
2. Jorge S, Jones NL, Chen L, Hou JY, Tergas AI, Burke WM, Ananth CV, Neugut AI, Herhshman DL and Wright JD: Characteristics, treatment and outcomes of women with immature ovarian teratoma, 1998-2012. Gynecol Oncol 142: 261-266, 2016.

3. Hung YC, Chang WC, Chen LM, Chang YY, Wu LY, Chung WM, Lin TY, Chen LC and Ma WL: Non-genomic estrogen/estrogen receptor $\alpha$ promotes cellular malignancy of immature ovarian teratoma in vitro. J Cell Physiol 229: 752-761, 2014.

4. Chan JK, Gardner AB, Chan JE, Guan A, Alshak M and Kapp DS: The influence of age and other prognostic factors associated with survival of ovarian immature teratoma-A study of 1307 patients. Gynecol Oncol 142: 446-451, 2016.

5. Eidem TM, Kugel JF and Goodrich JA: Noncoding RNAs: Regulators of the mammalian transcription machinery. J Mol Biol 428: 2652-2659, 2016.

6. Mattick JS and Makunin IV: Non-coding RNA. Hum Mol Genet 15 Spec No 1: R17-R29, 2006.

7. Esteller M: Non-coding RNAs in human disease. Nat Rev Genet 12: 861-874, 2011

8. Qi X, Zhang DH, Wu N, Xiao JH, Wang X and Ma W: ceRNA in cancer: Possible functions and clinical implications. J Med Genet 52: 710-718, 2015.

9. Liu D, Li Y, Luo G, Xiao X, Tao D, Wu X, Wang M, Huang C, Wang L, Zeng F and Jiang G: LncRNA SPRY4-IT1 sponges miR-101-3p to promote proliferation and metastasis of bladder cancer cells through up-regulating EZH2. Cancer Lett 388: 281-291, 2017.

10. Huang J, Chen YX and Zhang B: IGF2-AS affects the prognosis and metastasis of gastric adenocarcinoma via acting as a ceRNA of miR-503 to regulate SHOX2. Gastric Cancer 23: 23-38, 2019.

11. Livak KJ and Schmittgen TD: Analysis of relative gene expression data using real-time quantitative PCR and the 2(-Delta Delta C(T)) method. Methods 25: 402-408, 2001.

12. Franken NA, Rodermond HM, Stap J, Haveman J and van Bree C: Clonogenic assay of cells in vitro. Nat Protoc 1: 2315-2319, 2006.

13. Chang RK, Li X, Mu N, Hrydziuszko O, Garcia-Majano B, Larsson C and Lui WO: MicroRNA expression profiles in non-epithelial ovarian tumors. Int J Oncol 52: 55-66, 2018.

14. Ritchie ME, Phipson B, Wu D, Hu Y, Law CW, Shi W and Smyth GK: limma powers differential expression analyses for RNA-sequencing and microarray studies. Nucleic Acids Res 43: e47, 2015.

15. Zhu P, Wang Y, Wu J, Huang G, Liu B, Ye B, Du Y, Gao G, Tian Y, He L and Fan Z: LncBRM initiates YAP1 signalling activation to drive self-renewal of liver cancer stem cells. Nat Commun 7: 13608, 2016.

16. Salmena L, Poliseno L, Tay Y, Kats L and Pandolfi PP: A ceRNA hypothesis: The rosetta stone of a hidden RNA language? Cell 146: 353-358, 2011

17. Li N, Zeng J, Sun F, Tong X, Meng G, Wu C, Ding X, Liu L, Han M, Lu C and Dai F: p27 inhibits CDK6/CCND1 complex formation resulting in cell cycle arrest and inhibition of cell proliferation. Cell Cycle 17: 2335-2348, 2018.

18. Li Q and Lozano G: Molecular pathways: Targeting Mdm2 and Mdm4 in cancer therapy. Clin Cancer Res 19: 34-41, 2013.

19. Siddik ZH: Chapter 12-Apoptosis in cancer: Mechanisms, deregulation, and therapeutic targeting. In: Cancer Drug Design and Discovery. Neidle S (ed). 2nd edition. Academic Press, San Diego, CA, pp357-390, 2014.

20. Liu C, Kelnar K, Liu B, Chen X, Calhoun-Davis T, Li H, Patrawala L, Yan H, Jeter C, Honorio S, et al: The microRNA miR-34a inhibits prostate cancer stem cells and metastasis by directly repressing CD44. Nat Med 17: 211-215, 2011.

21. Zhao Y, Ponnusamy M, Zhang L, Zhang Y, Liu C, Yu W, Wang K and Li P: The role of miR-214 in cardiovascular diseases. Eur J Pharmacol 816: 138-145, 2017.

22. Zheng C, Guo K, Chen B, Wen Y and Xu Y: MiR-214-5p inhibits human prostate cancer proliferation and migration through regulating CRMP5. Cancer Biomark 26: 193-202, 2019.

23. Pang J, Li Z, Wang G, Li N, Gao Y and Wang S: MiR-214-5p targets KLF5 and suppresses proliferation of human hepatocellular carcinoma cells. J Cell Biochem: Sep 11, 2018 (Epub ahead of print).

24. Cao TH, Ling X, Chen C, Tang W, Hu DM and Yin GJ: Role of miR-214-5p in the migration and invasion of pancreatic cancer cells. Eur Rev Med Pharmacol Sci 22: 7214-7221, 2018.

25. Wu S, Gu Z, Wu Y, Wu W, Mao B and Zhao S: LINC00324 accelerates the proliferation and migration of osteosarcoma through regulating WDR66. J Cell Physiol 235: 339-348, 2020. 
26. Pan ZH, Guo XQ, Shan J and Luo SX: LINC00324 exerts tumor-promoting functions in lung adenocarcinoma via targeting miR-615-5p/AKT1 axis. Eur Rev Med Pharmacol Sci 22: 8333-8342, 2018.

27. Zou Z, Ma T, He X, Zhou J, Ma H, Xie M, Liu Y, Lu D, Di S and Zhang Z: Long intergenic non-coding RNA 00324 promotes gastric cancer cell proliferation via binding with HuR and stabilizing FAM83B expression. Cell Death Dis 9: 717, 2018.

28. VanArsdale T, Boshoff C, Arndt KT and Abraham RT: Molecular pathways: Targeting the cyclin D-CDK4/6 axis for cancer treatment. Clin Cancer Res 21: 2905-2910, 2015.
29. Jirawatnotai S, Hu Y, Livingston DM and Sicinski P: Proteomic identification of a direct role for cyclin d1 in DNA damage repair. Cancer Res 72: 4289-4293, 2012.

30. Shadfan M, Lopez-Pajares V and Yuan ZM: MDM2 and MDMX: Alone and together in regulation of $\mathrm{p} 53$. Transl Cancer Res 1 : 88-89, 2012. 